\title{
Volterra integral equations solved in Fredholm form using Walsh functions
}

\author{
W. F. Blyth R. L. May* $\quad$ P. Widyaningsih ${ }^{\dagger}$
}

(Received 8 August 2003; revised 6 Jan 2004)

\begin{abstract}
Recently Walsh function methods have been developed for the numerical solution of several classes of problems, mainly linear and nonlinear integral equations of both Volterra and Fredholm types. In addition, modifications of the basic approach have led to the solution of functional differential equations, variational problems and parameter estimation problems. Linear Volterra integral equations are rewritten as Fredholm integral equations with appropriately modified kernels. In this Fredholm equation form, the Walsh function solution method is more efficient than directly solving the Volterra equation. Walsh function methods are spectral methods but they have a natural grid interpretation. Multigrid methods and a variation on the use of Richardson extrapolation are used on six well known Volterra
\end{abstract}

${ }^{*}$ Dept. Mathematics \& Statistics, RMIT University, Melbourne, Australia. mailto:bill.blyth@rmit.edu.au; rob@rmit.edu.au

${ }^{\dagger}$ current address: Dept. of Mathematics, Universitas Sebelas Maret, Solo, IndonEsIA.

See http://anziamj.austms.org.au/V45/CTAC2003/Blyt for this article, (c) Austral. Mathematical Soc. 2004. Published May 14, 2004. ISSN 1446-8735 
test problems, re-written in Fredholm form, to illustrate that these methods provide effective and efficient solution methods.

\section{Contents}

1 Introduction

2 Walsh functions

3 Volterra integral equations and multigrid

4 Volterra equations as Fredholm equations

5 The double Walsh series for the kernel

C276

6 Solution using iteration and multigrid

7 Nested iteration and Richardson extrapolation

8 Conclusion

C280

References

C281

\section{Introduction}

New methods are always needed to solve integral equations because no single method works well for all such equations.

There has been considerable interest in solving differential and integral equations using techniques which involve Walsh functions $[2,3,4,10]$ and references therein. One of the motivations for these developments is that these methods usually involve the use of the fast Walsh Fourier transform, which 
is faster than most corresponding transforms such as the trigonometric fast Fourier transform. Also, Walsh functions appear to be easily incorporated into a wide variety of robust general purpose algorithms.

In [3] an effective method to solve linear Volterra integral equations problems was introduced and it was shown how multigrid and Richardson extrapolation methods can be applied to improve efficiency. The method is quite general and conceptually simple. However the direct solution of Volterra integral equations using Walsh function methods requires a degenerate kernel approach in that the first step is to separate the variables. Thus considerable preliminary work is needed before the Walsh series are introduced and the sum of terms increases the computational time required to set up the matrix equation that is to be solved by iteration for the Walsh coefficients.

In this paper we show that, by rewriting the problem in Fredholm form, the preliminary work and computational time (using the fast Walsh transform to obtain the double Walsh series for the modified kernel) to obtain the linear equation for the Walsh coefficients is greatly reduced. The efficiency of the solution of the equation for the Walsh coefficients can be improved successfully using multigrid and Richardson extrapolation methods. Thus Volterra equations can be solved effectively and efficiently with this new Walsh function method approach.

\section{Walsh functions}

The Walsh functions have many properties similar to those of the trigonometric functions. For example they form a complete, total collection of functions with respect to the space of square Lebesgue integrable functions. However, they are simpler in structure to the trigonometric functions because they take only the values 1 and -1 . They may be expressed as linear combinations of the Haar functions [8], so many proofs about the Haar functions carry over to the Walsh system easily. Moreover, the Walsh functions are Haar wavelet 
packets; see [11] for a good account of the properties of the Haar wavelets and other wavelets.

We use the ordering of the Walsh functions due to Paley [9]. Any function $f \in L^{2}[0,1)$ can be expanded as a series of Walsh functions

$$
f(x)=\sum_{i=0)}^{\infty} c_{i} W_{i}(x) \quad \text { where } \quad c_{i}=\int_{0}^{1} f(x) W_{i} d x .
$$

Fine [7] discovered an important property of the Walsh Fourier series: the $m=2^{n}$ th partial sum of the Walsh series of a function $f$ is piece-wise constant, equal to the $L^{1}$ mean of $f$, on each subinterval $[(i-1) / m, i / m]$. For this reason, Walsh series in applications are always truncated to $m=$ $2^{n}$ terms. In this case, the coefficients $c_{i}$ of the Walsh (-Fourier) series are given by

$$
c_{i}=\sum_{j=0}^{m-1} \frac{1}{m} W_{i j} f_{j},
$$

where $f_{j}$ is the average value of the function $f(x)$ in the $j$ th interval of width $1 / m$ in the interval $(0,1)$, and $W_{i j}$ is the value of the $i$ th Walsh function in the $j$ th subinterval. The order $m$ Walsh matrix, $\mathcal{W}_{m}$, has elements $W_{i j}$.

Let $f(x)$ have a Walsh series with coefficients $c_{i}$ and its integral from 0 to $x$ have a Walsh series with coefficients of $b_{i}$ : $\int_{0}^{x} f(t) d t=\sum_{i=0}^{\infty} b_{i} W_{i}(x)$. If we truncate to $m=2^{n}$ terms and use the obvious vector notation, then integration is performed by matrix multiplication $\mathbf{b}=P_{m}^{T} \mathbf{c}$ where

$$
P_{m}^{T}=\left(\begin{array}{cc}
P_{m / 2} & \frac{1}{2 m} I_{m / 2} \\
-\frac{1}{2 m} I_{m / 2} & O_{m / 2}
\end{array}\right), \quad P_{2}^{T}=\left(\begin{array}{cc}
\frac{1}{2} & \frac{1}{4} \\
-\frac{1}{4} & 0
\end{array}\right)
$$

and $I_{m}$ is the unit matrix, $O_{m}$ is the zero matrix (of order $m$ ), see [6]. 


\section{$3 \quad$ Volterra integral equations and multigrid}

Blyth et al. [2] introduced a Walsh function multigrid approach and Richardson extrapolation for two very simple Volterra integral equations:

$$
y(x)=1+x+\int_{0}^{x} y(t) d t \quad \text { and } \quad y(x)=\frac{2}{3}+\int_{0}^{x} y^{2}(t) d t .
$$

To be useful, this method was extended [3] to two classic test problems:

$$
y(x)=x+\int_{0}^{x} \sin (x-t) y(t) d t,
$$

with solution $y(x)=x+\frac{1}{6} x^{3}$ and

$$
y(x)=\cos x-\int_{0}^{x}(x-t) \cos (x-t) y(t) d t,
$$

with solution $y(x)=\frac{1}{3}(2 \cos \sqrt{3} x+1)$. These are degenerate kernel problems where the kernel can be written as a sum of terms with the variables separated. For example, equation (5) becomes

$$
y(x)=x+\sin x \int_{0}^{x} \cos t y(t) d t-\cos x \int_{0}^{x} \sin t y(t) d t .
$$

After developing some tools to multiply truncated Walsh series, these problems were rewritten by separating the variables, replacing all functions by their Walsh series and then multiplying and integrating as appropriate. The coefficients $c_{i}$ of the Walsh series for $y(x)$ were obtained by solving the resultant linear system by iteration. Multigrid methods and Richardson extrapolation were used to improve efficiency.

Since this method was successfully implemented for two test problems (with degenerate kernels), it can be implemented for most linear Volterra 
integral equations. This is the case since most problems are either of degenerate kernel type or can be approximated by a degenerate kernel problem.

Although the Walsh function method is a Galerkin method, in these cases it takes on some of the characteristics of degenerate kernel methods (see Atkinson [1, chapter 2]): it is quite general and conceptually simple. However, there is significant preliminary work to be done to obtain the matrix equation that is to be solved by iteration and to obtain an efficient algorithm.

\section{Volterra equations as Fredholm equations}

In order to avoid the preliminary work required (and the resulting sum of terms of products of Walsh series), instead of solving the Volterra equations as indicated above, they are rewritten as Fredholm integral equations, with a modified kernel $\tilde{K}(x, t)$, and solved in Fredholm form.

The first test problem (5) for example, can be rewritten in Fredholm form as

$$
y(x)=g(x)+\int_{0}^{1} \tilde{K}(x, t) y(t) d t,
$$

where $g(x)=x$ and

$$
\tilde{K}(x, t)= \begin{cases}\sin (x-t), & 0 \leq t<x \\ 0, & x \leq t<1\end{cases}
$$

The second test problem is equation (6).

Another four standard test problem Volterra integral equations were solved: 
Test problem 3 with solution $y(x)=\frac{1}{2}(\sin x+\sinh x)$ is

$$
y(x)=\sin x+\int_{0}^{x}(x-t) y(t) d t .
$$

Test problem 4 with solution $y(x)=e^{x}(1+x)^{2}$ is

$$
y(x)=e^{x}+2 \int_{0}^{x} \cos (x-t) y(t) d t .
$$

Test problem 5 with solution $y(x)=\frac{1}{2}\left(e^{x}+\cos x+\sin x\right)$ is

$$
y(x)=\cos x+\int_{0}^{x} y(t) d t .
$$

Test problem 6 with solution $y(x)=\frac{2}{\sqrt{5}} \sinh \left(\frac{\sqrt{5}}{2} x\right) e^{-x / 2}$ is

$$
y(x)=\sinh x-\int_{0}^{x} \cosh (x-t) y(t) d t .
$$

The Fredholm integral equations form of these test problems is obvious and so details are omitted here. They are all of the form of (8). To solve a Volterra problem in the Fredholm form, Blyth and Uljanov [4] represent functions $y$ and $g$ by their Walsh series, truncated to $m$ terms. The kernel, $\tilde{K}(x, t)$, is approximated by a truncated double Walsh series

$$
\tilde{K}(x, t)=\sum_{i=0}^{m-1} \sum_{j=0}^{m-1} k_{i j} W_{i}(x) W_{j}(t),
$$

where

$$
k_{i j}=\int_{0}^{1} \int_{0}^{1} \tilde{K}(x, t) W_{i}(x) W_{j}(t) d x d t
$$




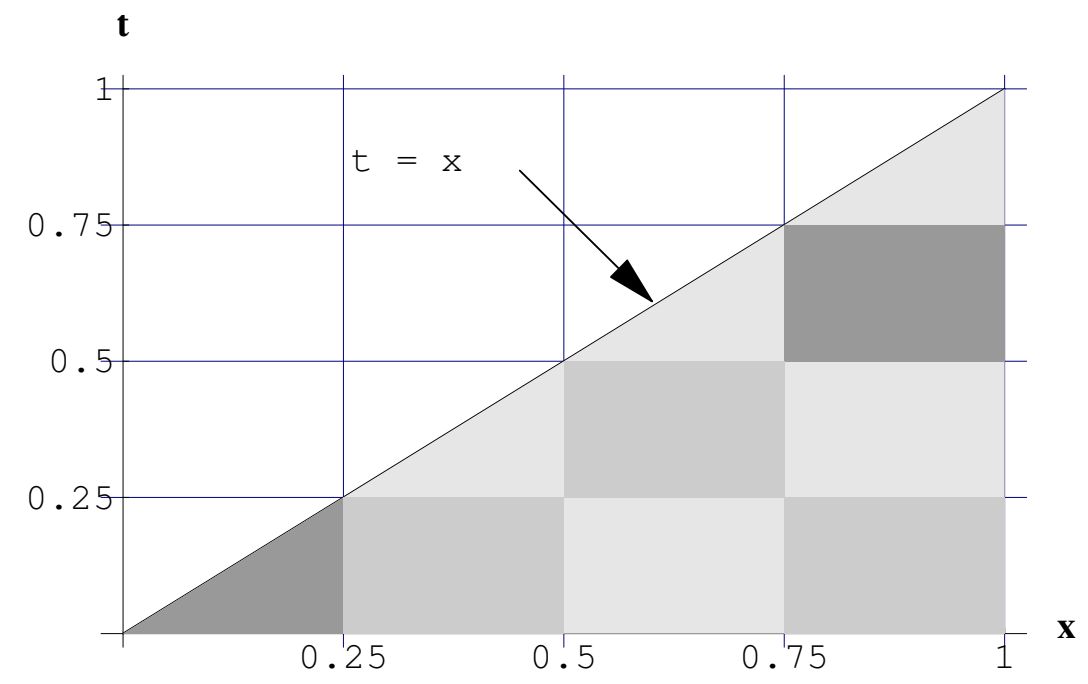

Figure 1: an illustration of the subintervals for $m=4$.

and it is easy to show that the integral equation can be rewritten as the simple linear problem

$$
\mathbf{c}_{m}=\mathbf{g}_{m}+K_{m} \mathbf{c}_{m}
$$

where $K_{m}$ is the matrix with elements $k_{i j}$. This was solved directly, but here we solve this linear problem (11) by ordinary (Picard) iterations and then investigate the use of the multigrid technique and Richardson extrapolation to improve efficiency.

\section{The double Walsh series for the kernel}

The first task is to calculate the matrix containing the coefficients of double Walsh series $K_{m}=\left(k_{i j}\right)$. If $m=2^{n}$ (as is always the case in applications), then $K_{m}=\frac{1}{m^{2}} \mathcal{W}_{m} \bar{K}_{m} \mathcal{W}_{m}$. This is the double Walsh transform of the matrix $\bar{K}_{m}$ of the average values of $\tilde{K}(x, t)$ on all subintervals, see Figure 1. 
Since the area of each of these $2 \mathrm{D}$ subintervals is $1 / \mathrm{m}^{2}$, we rewrite this as

$$
K_{m}=\mathcal{W}_{m} A_{m} \mathcal{W}_{m}
$$

where $A_{m}$ is the matrix of the double integrals of $\tilde{K}(x, t)$ over the subintervals. For the $i$ th $\mathrm{x}$-subinterval and $j$ th $t$-subinterval of a square subregion, the element of $A_{m}$ is

$$
a_{i j}=\int_{(i-1) / m}^{i / m} \int_{(j-1) / m}^{j / m} \tilde{K}(x, t) d t d x, \quad i>j .
$$

For $a_{i i}$ the modified kernel is non zero only on a triangular subregion, so the upper limit of the inner integral must be changed to $x$.

The matrix $A_{m}$ is constructed for the finest grid, that is the largest $m$ (equal to 512 here), and then elements are added to give $A_{m}$ for the coarser grids down to $m=4$. The matrix $K_{m}$ is calculated for the largest $m$ and successively truncated as $m$ is halved.

\section{Solution using iteration and multigrid}

Equation (11) can be solved for the vector $\mathbf{c}_{m}$ of coefficients by standard Picard iteration. Since the exact solution is known, then $\mathbf{c}_{\text {Exact }}$ can be calculated. The iterations $\mathbf{c}_{m}^{[i]}$ do not converge to $\mathbf{c}_{\text {Exact }}$ but to $\mathbf{c}_{\text {Trunc: } m}$ (the exact solution of (11) for a given $m$ ).

For given $m$, the $i$ th iteration error is defined as $\left\|\mathbf{c}_{\text {Trunc: } m}-\mathbf{c}_{m}^{[i]}\right\|$ and the truncation error as $\left\|\mathbf{c}_{\text {Exact }}-\mathbf{c}_{\text {Trunc: } m}\right\|$. In our error analysis, the Euclidean norm is used. This is a natural choice since the Walsh series are generalized Fourier series with the Walsh functions forming a complete and orthonormal set of basis functions. Thus, from Parseval's identity, the $L^{2}$ norm of a function is the $l^{2}$ norm of the Walsh coefficients. For the test problems, 
solved via Equation (11), an error tolerance of $10^{-8}$ (for the iteration error) was used for $m=512$. Test problems 1 to 6 required 6, 5, 6, 14, 10, and 11 (respectively) Picard iterations.

To improve efficiency, Blyth et al. [2,3] incorporated multigrid techniques. These are easy to implement even though Walsh function methods are spectral methods, since they are naturally associated with a grid where the unit interval is divided into $m=2^{n}$ equal width subintervals. For an introduction to multigrid techniques, see Briggs [5].

Information from the coarse grid, corresponding to a Walsh series with $\mathrm{m} / 2$ coefficients, is transferred (a process called prolongation) to a fine grid (of half the grid spacing - corresponding to using Walsh series with $m$ terms). For the $m$ Walsh coefficients on the fine grid, the first $m / 2$ Walsh coefficients are set equal to the $m / 2$ coefficients on the coarse grid and the remaining $m / 2$ coefficients zero.

Similarly vectors (of Walsh coefficients) from the fine grid (with $m$ elements) are transferred to the coarse grid (by "restriction") by discarding the last $m / 2$ elements.

We choose to use $\mathrm{V}$ cycles with one iteration at each level at the top, going down each level and going back up again as well as the top level. So, from $m=512$, a 2-level V cycle, $\mathrm{V}_{2}$, does one iteration for $m=512$, computes a residual and transfers this (by restriction) to the $m=256$ level where the residual correction is computed (on the coarser grid). This residual correction is transferred to the fine grid with $m=256$ (by prolongation) which is corrected and one iteration is done with this corrected vector on the fine grid. This takes about $2 \frac{1}{4}$ "Work Units", wU's, which are Picard iterations at the finest grid.

The solution of the first test problem is typical of the first three problems. With an error tolerance of $10^{-8}$ (for the iteration error), 6 Picard iterations for $m=512$ or two 2-level $\mathrm{V}$ cycles (with 4.5 wU's) are required. This is the best choice for efficiency (since the use of extra levels does not give much 
improvement but does increase the computational cost. The observation that the best choice for efficiency is to use $\mathrm{V}$ cycles of (only) 2 levels is consistent with experience of many implementations of multigrid methods, see [1].

Test problem 4 has the slowest convergence - 14 Picard iterations or $4 \mathrm{~V}_{2}$ (9 WU's) or $3 \mathrm{~V}_{3}$ (7.7 WU's) or $2 \mathrm{~V}_{4}$ (5.3 WU's) are required. The results for the test problems 5 and 6 are very similar where the error tolerance is achieved with 10 and 11 (respectively) Picard iterations or $3 \mathrm{~V}_{2}$ (6.75 WU's) or $2 \mathrm{~V}_{3}$ (5.1 WU's) or $2 \mathrm{~V}_{4}$ (5.3 WU's). For these more slowly convergent problems, the best choice for efficiency is to use $\mathrm{V}$ cycles of (slightly) more than 2 levels.

\section{Nested iteration and Richardson extrapolation}

Multigrid methods increase efficiency but work with an error tolerance that is the iteration error - not the actual (total) error. Since Walsh function methods are locally convergent with order two, we have introduced Richardson extrapolation [2,3]. This idea is applied in two ways. The final result is extrapolated (in the usual way) to estimate the "exact" result. This uses the familiar formula where $\mathbf{c}_{\text {Exact }}$ is approximated by $\frac{4}{3} \mathbf{c}_{2 m}-\frac{1}{3} \mathbf{c}_{m}$.

To estimate good coefficients for the next grid when using nested iteration, we consider the two previous (coarse) grid levels and use Richardson extrapolation to estimate the Walsh coefficients for the next level: $\mathbf{c}_{4 m} \approx \frac{5}{4} \mathbf{c}_{2 m}-\frac{1}{4} \mathbf{c}_{m}$.

Of course the estimation of $\mathbf{c}_{4 m}$ can be applied only for the first $m$ of the $4 m$ terms. For further details, see [2,3]. We call this a Richardson seed (for the iteration at the $4 m$ level). Our Richardson seed nested iteration method starts by calculating the coefficients of $m=4$ and $m=8$ by the ordinary iteration in that level using 6 iterations. From $m=16$ and upwards, 
TABLE 1: Iteration error and total error for $m=512$ using Richardson seed and nested iteration for test problems 1 and 4 .

\begin{tabular}{|cc|cc|cc|}
\hline & & \multicolumn{2}{|c|}{ Test problem 1 } & \multicolumn{2}{c|}{ Test problem 4 } \\
$\nu$ & WU & Iteration Error & Total error & Iteration Error & Total error \\
\hline 1 & 1.33 & $6.01 \times 10^{-8}$ & $6.56 \times 10^{-10}$ & $1.78 \times 10^{-4}$ & $5.88 \times 10^{-4}$ \\
2 & 2.67 & $2.30 \times 10^{-9}$ & $3.74 \times 10^{-10}$ & $1.56 \times 10^{-5}$ & $6.45 \times 10^{-6}$ \\
3 & 4.00 & $4.71 \times 10^{-11}$ & $3.65 \times 10^{-10}$ & $7.39 \times 10^{-6}$ & $6.56 \times 10^{-8}$ \\
4 & 5.33 & $5.83 \times 10^{-13}$ & $3.65 \times 10^{-10}$ & $3.35 \times 10^{-6}$ & $5.21 \times 10^{-8}$ \\
5 & 6.67 & $4.82 \times 10^{-15}$ & $3.65 \times 10^{-10}$ & $1.32 \times 10^{-6}$ & $4.52 \times 10^{-8}$ \\
\hline
\end{tabular}

we perform nested iteration with our Richardson seed and $\nu$ iterations at each level, up to the highest $(m=512)$ level. At the finest grid, ordinary Richardson extrapolation is used to estimate the solution. The error of this extrapolated solution is called the total error.

Test problem 1 (typical of the first three test problems) has truncation error $2.4 \times 10^{-7}$ and Table 1 shows that one iteration, $\nu=1$, gives high accuracy for the total error. Using $\nu=2$ gives a small improvement; more iterations reduce the iteration error but not the total error.

Test problem 4 (which has the slowest convergence), gives high accuracy for the total error with $\nu=3$, see Table 1 . This takes only 4 wU's. More iterations reduce the iteration error but don't reduce the total error by much.

\section{Conclusion}

The direct solution of Volterra integral equations using Walsh function methods requires a degenerate kernel approach in that the first step is to separate the variables. Thus considerable preliminary work is needed before the Walsh series are introduced and the sum of terms increases computational time required to set up the linear equation, corresponding to (11). 
By rewriting the problem in Fredholm form, the computational time to obtain the linear equation (11) is greatly reduced. For simplicity, we used the double Walsh transform (12) here, but in practice the fast Walsh transform, FWT, would be used. The FWT is an analogue of the fast Fourier transform, but is faster.

Multigrid methods can be used to improve efficiency. However our "work unit" measure ignores (as is usually the case [5]) some overheads such as inter-grid transfers. We also ignore the cost of calculation of the residual, so this would add half a wU for each 2-level V cycle. However multigrid methods reduce iteration error and only slightly effect the total error. We conclude that out Richardson seeded nested iteration method, with extrapolation, is superior: it is efficient and it reduces the total error. The Walsh function method to solve Volterra equations is fast and efficient when our Richardson seeded nested iteration method is applied to the Fredholm form of the problem.

\section{References}

[1] K. E. Atkinson. The Numerical Solution of Integral Equations of the Second Kind. Cambridge University Press, 1997. C274, C279

[2] W. F. Blyth, R. L. May and P. Widyaningsih. The Solution of Integral Equations Using Walsh Functions and a Multigrid Approach. In J. Noye et al., editors, Computational Techniques and Applications: CTAC97, Proceedings, 99-106. World Scientific, Singapore, 1998. C270, C273, C278, C279

[3] W. F. Blyth, R. L. May and P. Widyaningsih. Solution of Separable Volterra Integral Equations Using Walsh Functions and a Multigrid Approach. In M. Pemberton, I. Turner and P. Jacobs, editors, Engineering Mathematics and Applications Conference: EMAC 2002 
Proceedings, 85-90. The Inst. of Engineers, Australia, 2002. C270, C271, C273, C278, C279

[4] W. F. Blyth and V. Uljanov. Numerical Solution of Weakly Singular Fredholm Integral Equations using Walsh Functions. In R. L. May and A. K. Easton, editors, Computational Techniques and Applications: CTAC-95, Proceedings, 137-143. World Scientific, Singapore, 1996. $\mathrm{C} 270, \mathrm{C} 275$

[5] W. L. Briggs. A Multigrid Tutorial. SIAM, Philadelphia, 1987. C278, $\mathrm{C} 281$

[6] C. F. Chen and C. H. Hsiao. A Walsh series direct method for solving variational problems. J. Franklin Inst., 300, 4, 265-280, 1975. C272

[7] N. J. Fine. On the Walsh Functions. Trans. Amer. Math. Soc., 65, 372-414, 1949. C272

[8] A. Haar. Zur Theorie der orthogonalen Funktionen systeme. Math. Ann., 69, 331-371, 1910. C271

[9] R. E. A. C. Paley. A remarkable series of orthogonal functions (I \& II). London Math. Soc., 34, 241-264 \& 265-279, 1932. C272

[10] B. G. Sloss and W. F. Blyth. A Walsh function method for a nonlinear Volterra integral equation. J. Franklin Inst., 6, 239-255, 1994. C270

[11] P. Wojtaszczyk. A mathematical introduction to wavelets. Cambridge University Press, 1997. C272 